\title{
Evaluación de las transformaciones estructurales en recubrimientos de WC10Ni depositados por laser cladding sobre acero para herramienta EN 12379(*)
}

\author{
J.J. Candel*, V. Amigó, J. Sampedro** y V. Bonache*
}

Resumen

\begin{abstract}
Los materiales compuestos de matriz metálica reforzados con carburos, son conocidos por su elevada resistencia a todos los tipos de desgaste, debido a la combinación de las partículas duras en una matriz metálica tenaz. Diferentes tipos de estos materiales, se han empleado en el desarrollo de nuevas herramientas de corte de altas prestaciones. La técnica de láser cladding (LC), permite obtener recubrimientos libres de defectos sobre zonas muy concretas, con un aporte de calor muy localizado. Pero en el caso de carburos de wolframio (WC), debido a la enorme absorción de energía y la diferencia de propiedades entre el metal base y la cerámica, puede producir una gran cantidad de defectos tales como grietas, poros, gran dilución de carburos, falta de adherencia, etc. El objetivo de este trabajo, es estudiar las transformaciones metalúrgicas que aparecen durante el procesado por láser de recubrimientos tipo cermet de WC10Ni, sobre acero de herramienta para trabajo en frío (EN 12379). Además se ha relacionado los parámetros de proceso con la generación de defectos. Para ello, se analiza su microestructura, composición y se obtienen perfiles de dureza en el recubrimiento y en el acero afectado por el calor. Los resultados muestran, que aunque el control de los parámetros del proceso reduce la generación de defectos, al depositar recubrimientos por solape de cordones, se produce gran cantidad de transformaciones debido a la disolución masiva de las partículas de WC y la difusión de elementos de aleación, desde el sustrato hacia el recubrimiento.
\end{abstract}

\section{Evaluation of structural changes in WC10Ni coatings deposited by laser cladding on tool steel EN 12379}

\begin{abstract}
Carbide metal matrix composite materials are known for a high resistance to all types of wear. It is due to a beneficial combination of properties given by hard phase particles included in a tough matrix. Different kinds of those materials have been employed in the development of new high properties cutting tools. Laser cladding (LC) technique allows obtaining an accurate defect-free coating with a low thermal affectation of the component. But in the case of WC cermet coatings due to its high laser absorption and the different mechanical and thermal properties between substrate and coating can appear a wide range of different defects as cracks, pores, massive carbide dilution and lacks of adherence. The aim of the present work is to study the metallurgical transformations during LC process of WC cermet coating on cold work tool steel substrate (EN 12379). Also it has been related process parameters with defects generation. Microstructure and composition of the coating and the heat affected zone have been analysed. Microhardness evolution profile has been obtained. Results show that although process parameters control reduce the generation of defects, in the deposition of overlapped layers appear different metallurgical transformations related with massive WC decomposition and the diffusion of alloying elements from substrate to the coating.
\end{abstract}

Keywords

Laser cladding; Tool steel; WC cermet; Microstructure.

\section{INTRODUCCIÓN}

Los materiales compuestos de matriz metálica basados en carburo de wolframio (WC), son conocidos por su combinación de propiedades de alta tenacidad y resis- tencia mecánica, así como elevada dureza y resistencia frente al desgaste. Por este motivo, se ha trabajado mucho para conseguir recubrimientos de WC con diferentes matrices metálicas por proyección térmica. Así se podría mejorar superficialmente muchos com-

\footnotetext{
(•) Trabajo recibido el día 03 de Septiembre de 2009 y aceptado en su forma final el día 11 de Agosto de 2010.

* Instituto de Tecnología de Materiales, Universidad Politécnica de Valencia, España. E-mail: vamigo@mcm.upv.es

** Instituto Tecnológico de Óptica, Color e Imagen (AIDO) - Paterna, España.
} 
ponentes estructurales. De entre todos los materiales estudiados, destacan los recubrimientos compuestos de base níquel ya que poseen una elevada resistencia a la oxidación ${ }^{[1]}$.

En especial, en los recubrimientos de WC por proyección térmica de alta velocidad (HVOF), aparecen problemas de porosidad y falta de adherencia tanto entre el recubrimiento y el substrato como entre partículas proyectadas. Para superar estos problemas, se ha desarrollado aleaciones de $\mathrm{NiCrBSiFe}$ WC con propiedades autofundentes que mejoran las propiedades y reducen los defectos. Estas aleaciones son capaces de formar eutécticos, con un fuerte endurecimiento por precipitación de compuestos cuando contienen pequeñas cantidades de boro, silicio y hierro, además de poseer una excelente resistencia a la corrosión ${ }^{[2]}$.

La proyección por HVOF, si bien presenta una alta productividad, no es adecuada para recubrir selectivamente pequeñas zonas de útiles ni para reparar componentes superficialmente. En este caso, la reparación por láser cladding (LC), puede ser una alternativa muy interesante, ya que permite obtener recubrimientos duros y libres de defectos en muchas aplicaciones, con una afectación mínima del substrato $^{[3]}$.

Las ventajas del procesado por láser, debidas a la alta concentración de energía, en la práctica suponen muchos inconvenientes para la obtención de recubrimientos de metal duro por laser cladding. Las partículas de WC se disuelven y precipitan en la matriz metálica. De manera que, cuando la cantidad de WC disuelto es alta, aparecen grandes lagunas extremadamente duras y frágiles que no soportan el rápido enfriamiento impuesto por este calentamiento tan localizado y provocan el agrietamiento del recubrimiento. Incluso con cantidades de refuerzo inferiores al $50 \%$ en peso, pueden aparecer estos problemas si no se realiza un control preciso de los parámetros de proceso ${ }^{[4-6]}$.

Si se define la energía aportada como $P /(V \cdot D)$, siendo $P$ la potencia del láser, $V$ la velocidad de avance y $D$ el diámetro del haz. $\mathrm{Al}$ aumentar la energía aportada disminuye la cantidad de partículas no fundidas en el recubrimiento y baja la velocidad de enfriamiento. Pero en el caso de los recubrimientos con WC, debido a su baja conductividad térmica y fuerte absorción de la radiación láser, se produce la dilución masiva del carburo y se forman fases frágiles no deseadas. Además, el aumento de la energía aumenta la afectación térmica del substrato y favorece la difusión de elementos de aleación, entre el substrato y el recubrimiento. Por tanto, elementos como el cromo, silicio, hierro y vanadio, presentes en muchos aceros para herramientas pueden incor- porarse al recubrimiento y formar estructuras de

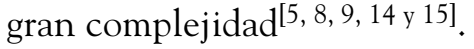

El objetivo de este trabajo, es analizar la microestructura y evaluar los fenómenos de difusión que aparecen durante el proceso de reparación por laser cladding (LC), de un acero para herramienta EN 12379, con un alto contenido en cromo y otros elementos de aleación, mediante polvos de WC base níquel.

\section{TRABAJO EXPERIMENTAL}

\subsection{Equipo láser empleado}

Las deposiciones han sido realizadas con un sistema láser de Nd:YAG (Trumpf HL1006D), de $1 \mathrm{KW}$ de potencia máxima en modo continuo. La propagación del haz láser, se produce a través de una fibra óptica de 0,6 $\mathrm{mm}$ de diámetro en el núcleo. El sistema de focalización está compuesto por un colimador con una focal de $200 \mathrm{~mm}$, que proporciona un diámetro del haz en el foco de 0,6 $\mathrm{mm}$. La experimentación se ha llevado a cabo desenfocando el haz láser, hasta obtener una haz de 2,5 $\mathrm{mm}$ aproximadamente, medido con un analizador de haz (Beam Monitor, Primes GMBH), mediante el método del $86 \%$ para todas las potencias experimentadas.

El perfil de irradiancia del haz a la distancia de desenfoque, se corresponde a un perfil aproximadamente gaussiano por degradación del "top hat", que se genera en el foco.

El polvo fue depositado mediante un alimentador de polvo Sulzer Twin 10C a través de una boquilla coaxial. El gas empleado para proteger las deposiciones realizadas, ha sido argón.

El movimiento de la pieza, se consigue mediante una mesa de ejes cartesianos X-Y-Z, controlada por un control numéricos SINUMERIC con una precisión de $0,01 \mathrm{~mm}$.

\subsection{Materiales empleados}

De entre todas las aleaciones férreas para trabajo en frío (menos de $200^{\circ} \mathrm{C}$ ), se ha elegido el acero EN 12379 (AISI D2), con un tratamiento térmico específico de temple y revenido, de manera que se simule el proceso real de reparación de una pieza.

Estos aceros poseen una elevada dureza, debido a la formación de carburos complejos de cromo y vanadio en una matriz de tipo martensítico, lo que permite obtener durezas por encima de $60 \mathrm{HRc}$, con el tratamiento térmico adecuado. La composición química 
del acero empleado es la siguiente: $1,6 \% \mathrm{C} ; 1,0 \% \mathrm{Si}$; 0,3 \% Mn; $11 \% \mathrm{Cr} ; 0,8 \%$ Mo y 0,8 \% V.

Como material base se han empleado probetas planas metálicas de $5 \mathrm{~mm}$ de espesor, cortadas en formatos de $100 \times 150 \mathrm{~mm}$. Las probetas se han templado en el acero a $1.020-1.040{ }^{\circ} \mathrm{C}$ para conseguir la disolución de los carburos seguido de un enfriamiento en aceite. Finalmente, se aplicó un revenido para eliminar tensiones y formar una segunda precipitación de carburos, que aumenta la dureza útil a $500{ }^{\circ} \mathrm{C}$ durante $2 \mathrm{~h}$.

Como material de aporte, se ha empleado polvo de WC10Ni con tamaño de partícula comprendido entre $20-45 \mu \mathrm{m}$, suministrado por Sulzer-Metco (Woka3302®).

\subsection{Recubrimientos obtenidos}

Se han depositado cordones de $30 \mathrm{~mm}$ de longitud con diferentes condiciones de potencia, velocidad de avance y caudal de polvo. Se depositaron dos series de muestras, para encontrar las mejores condiciones de trabajo posibles. En la primera, se realizó un amplio barrido de las condiciones de proceso que permite el equipo. La segunda, se centró en los parámetros que producen mejores resultados (Tabla 1 ).

En ambas series, la distancia de la boquilla al plano de trabajo ha sido de $12 \mathrm{~mm}$ y se empleó argón como gas de proceso con un caudal de $4 \mathrm{l} / \mathrm{min}$. De entre todos los cordones, se han elegido aquellos que tenían menor cantidad de defectos, (poros y grietas) y se realizaron recubrimientos formados por 12 cordones solapados a $0,7 \mathrm{~mm}$, para evaluar las transformaciones durante un proceso de reparación real. Se han identificado como recubrimiento 1 y 2 .

\subsection{Caracterización microestructural}

Se ha realizado cortes transversales con una cortadora de precisión con disco de diamante, de los cordones de cada plantilla y de los recubrimientos obtenidos por solape. A continuación se realizaron las preparaciones metalográficas, mediante desbaste con papel de lija de $\mathrm{SiC}$ y pulido con pasta de diamante. El metal base se atacó con Nital - 3 (3\% de $\mathrm{HNO}_{3}$ ).

Para el análisis microestructural se ha empleado microscopia óptica con un equipo Nikon Microphot FX y con microscopia electrónica de barrido (SEM), con un equipo JEOL 6300 trabajando en modo de electrones secundarios (SE) y retrodispersados (BSE). Se ha realizado análisis químico semicuantitativo, mediante sonda de Energías dispersivas de Rayos X (EDX) acoplada al SEM.

La caracterización mecánica de los recubrimientos, se ha llevado a cabo mediante medidas de microdureza Vickers en diferentes zonas del recubrimiento, con un equipo DURAMIN-1, aplicando una carga de $500 \mathrm{~g}$ durante $10 \mathrm{~s}\left(\mathrm{HV}_{0,5}\right)$. Se ha estudiado tres zonas diferentes, promediándose 3 medidas separadas de $50 \mu \mathrm{m}$ entre sí, una en la parte superior del cordón, la segunda en la parte inferior del cordón y la tercera en la zona de dilución en el acero de herramienta.

\section{RESULTADOS Y DISCUSIÓN}

\subsection{Formación de defectos}

La caracterización de las pruebas realizadas en la primera serie, muestra que hay una gran cantidad de condiciones que producen recubrimientos defectuosos. La penetración del cordón es excesiva, se forman

Tabla 1. Mapa de las deposiciones realizadas. La serie 1 se ha barrido todo el rango de potencias y velocidades. Una vez analizados los resultados, se ha centrado la ventana de proceso en los parámetros más adecuados

Table 1. Deposition map. In the batch 1 has been investigated the whole range of powers and speeds. After analyzing the results, the process window has been focused on the best settings

\begin{tabular}{ccccc}
\hline & $\begin{array}{c}\mathbf{P} \\
\mathbf{( W )}\end{array}$ & $\begin{array}{c}\mathbf{V} \\
(\mathbf{m} / \mathbf{m i n})\end{array}$ & $\begin{array}{c}\mathbf{M} \\
(\mathbf{g} / \mathbf{m i n})\end{array}$ & $\begin{array}{c}\mathbf{D} \\
(\mathbf{m m})\end{array}$ \\
\hline Serie 1 & $400-1000$ & $0,25-0,75$ & $3-9$ & 1,5 \\
Serie 2 & $300-500$ & $0,25-0,5$ & 3 & 2 \\
Recubrimiento 1 & 400 & 0,25 & 5 & 2 \\
Recubrimiento 2 & 500 & 0,375 & 8 & 2 \\
\hline
\end{tabular}


muchos poros de gran tamaño y prácticamente la totalidad de recubrimientos presentan grietas (Fig. 1 A)). Las imágenes obtenidas mediante MEB de electrones retrodispersados, muestra una microestructura de solidificación, donde prácticamente todos los carburos se han fundido durante el procesado y han precipitado durante el enfriamiento, formando microestructuras dendríticas de gran complejidad (Fig. 1 B)).

El análisis mediante EDX de los compuestos formados, indica que su composición en peso (detalle B1, Fig. 1 B)) es 42,2 \% W; 28,73\% Fe; 16,86 \% Cr; $8,68 \% \mathrm{Ni} ; 3,53 \%$ C. Según las tipologías identificadas por Riabkina et al. ${ }^{[11]}$, se trata de estructuras con alto contenido en wolframio, en las que se han incluido elementos de aleación como el cromo y el hierro, que contribuyen a formar complejas estructuras de tipo eutéctico. En estos casos, aunque se evite la defectología macroscópica (poros y grietas), este tipo de estructura no es adecuada ya que su comportamiento es frágil.

Se ha comprobado, en concordancia con los resultados de 1, 4 y 5, que la potencia del láser (P) y la velocidad de avance de la masa $(\mathrm{V})$, tienen una gran influencia en la dilución de carburos y formación de grietas por desgarros en caliente durante la deposi-

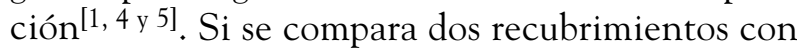
diferentes energía de la serie 1 (Figs. 1 A) y 1 C)),

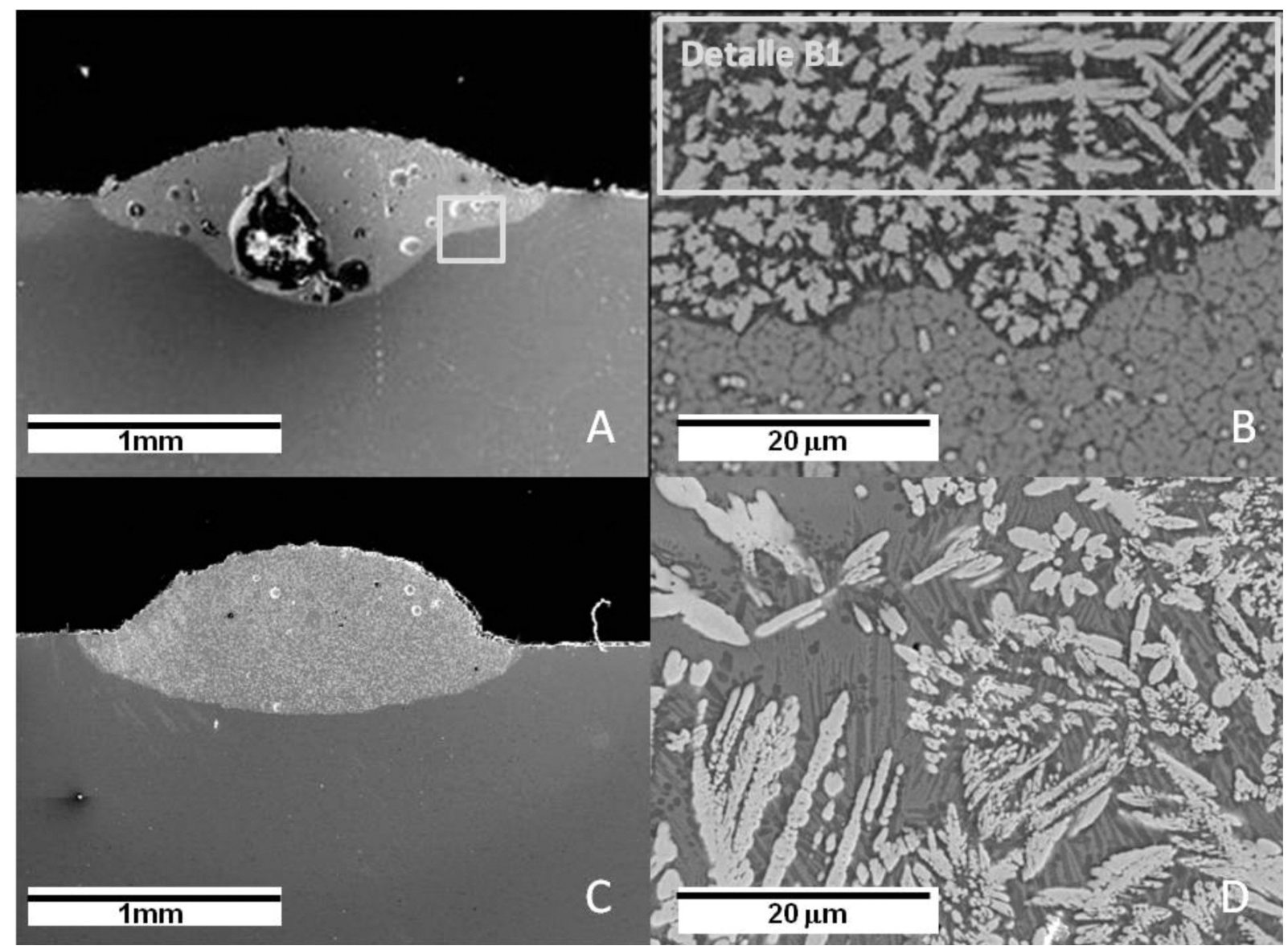

Figura 1. Imagen general (A) y microestructura (B) de un cordón de WC10Ni de la serie $1(P=700 \mathrm{~W}, V=500 \mathrm{~mm} / \mathrm{min})$. Se aprecia la formación de defectos internos y la fusión completa del WC, debido a la acumulación de calor en el interior del cordón. Mediante la reducción de la potencia y velocidad $(P=600 \mathrm{~W}, \mathrm{~V}=400 \mathrm{~mm} / \mathrm{min})$, se consiguió eliminar las grietas y poros (C), pero no se evitó la fusión de los carburos (D).

Figure 1. General view (A) and microstructure (B) of a layer WC10Ni Series $1(P=700 \mathrm{~W}, V=500 \mathrm{~mm} / \mathrm{min})$. It can be seen the formation of internal defects and complete melting of the WC due to heat accumulation inside the layer. By reducing the power and speed $(P=600 \mathrm{~W}, V=400 \mathrm{~mm} / \mathrm{min}$ ) was achieved a cracks and pores reduction $(C)$, but could not be avoided the fusion of the carbides (D). 
se observa que disminuyen los poros y grietas, aunque no se consiguió evitar la dilución de los carburos. Este hecho está relacionado con la baja entalpía de formación del WC de $38,5 \mathrm{KJ} / \mathrm{mol}$, que produce que el láser diluya gran cantidad de carburos dentro del baño fundido, tal y como destaca $\mathrm{Wu}^{[16]}$, formándose lagunas de dilución con una gran fragilidad, debido a la aparición de carburos secundarios precipitados durante el enfriamiento.

Sin embargo, la formación de poros y la formación de aglomerados de carburos en la zona inferior del cordón, está relacionada con la ineficiencia de las fuerzas de Marangoni, para distribuir correctamente el material en el baño ${ }^{[1,7 \text { y } 8]}$. El parámetro de proceso con mayor influencia en este fenómeno, es la velocidad de enfriamiento, condicionada por la velocidad de avance de la pieza $V$; a mayor velocidad, mayor cantidad de porosidad ${ }^{[9]}$. Así pues, es necesario un estricto control de la energía aportada, para controlar la microestructura del recubrimiento y por ese motivo se ha realizado una segunda serie con menores energías aportadas.

El objetivo de esta segunda serie es evitar la fusión de los carburos primarios, manteniendo su forma cúbica facetada y por ello es necesario reducir la potencia.

Pero para conseguir mantener la adherencia y evitar el agrietamiento, se puede optar por precalentar el substrato o bien por reducir la velocidad de avance. En este trabajo, no se contaba con la posibilidad de precalentar, por lo que se ha recurrido a reducir la velocidad de avance. Para obtener cordones con la forma y tamaño de los de la primera serie, es necesario mantener el ratio $F / V$ siendo $F$ la cantidad de polvo soplado en $\mathrm{g} / \mathrm{min}$. Por lo que se ha reducido también la cantidad de polvo soplado.

En la segunda serie se ha podido comprobar que el control de la energía aportada, produce una reducción de la cantidad de macrodefectos. A modo de ejemplo, se muestra una imagen general de la deposición a $\mathrm{P}=400 \mathrm{~W}, \mathrm{~V}=250 \mathrm{~mm} / \mathrm{min}, \mathrm{M}=3 \mathrm{~g} / \mathrm{min}$ (Fig. 2).

Sin embargo, al emplear estos parámetros para generar recubrimientos por solape el material se

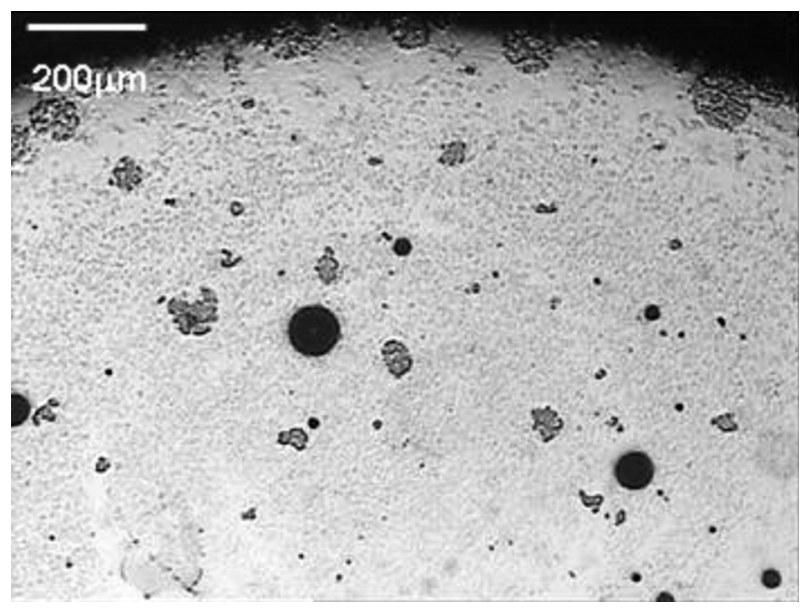

Figura. 2. Imagen general del cordón a $P=400 \mathrm{~W}$, $V=0,25 \mathrm{~mm} / \mathrm{min}$. Se observa como se ha conseguido mejorar el aspecto visual de la deposición.

Figure 2. General view of the layer at $P=400 \mathrm{~W}$, $V=0.25 \mathrm{~mm} / \mathrm{min}$. It can be seen an improvement of the visual aspect of the deposition.

comporta de manera distinta. Ya durante el procesado, se detectó un incremento progresivo de la cantidad de plasma y proyecciones sólidas desde el cordón, conforme se solapan más cordones. La inspección visual muestra que los recubrimientos tienen un aspecto irregular, con porosidad y grietas en su interior, tal y como confirman las figuras 3 y 4 . Las grietas crecen verticalmente en el recubrimiento y progresan a través de los poros hasta el substrato.

Es importante destacar, que los valores elegidos, se encuentran dentro de la ventana de parámetros empleada por otros autores, como $\operatorname{Lim}^{[7]}$ y Wang ${ }^{[8]}$, aunque en este caso se emplea un polvo con una fracción en peso de WC mayor. De acuerdo con Wang, estos defectos son inevitables en los recubrimientos tipo cermet sin precalentamiento.

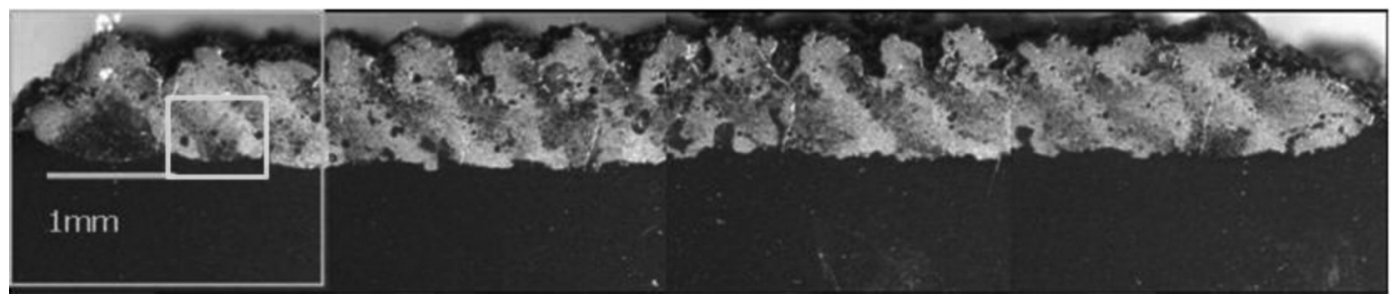

Figura 3. Sección transversal del recubrimiento 1.

Figure 3. Cross section of coating 1. 
Al comparar los recubrimientos obtenidos mediante imágenes en microscopía óptica, se comprueba que hay grandes diferencias entre todos los cordones de cada recubrimiento. En general, reducir la velocidad de avance (recubrimiento 1), reduce la cantidad de porosidad y agrietamiento, aunque con una mayor dilución de los carburos (lagunas de color más claro), debido a la acumulación del calor durante la deposición del recubrimiento, aunque se compense con la potencia aportada (Fig. 4). Dichas lagunas se forman mayoritariamente en la parte inferior del recubrimiento y en los solapes entre cordones.

No obstante, debe destacarse que ambos recubrimientos presentan una gran discontinuidad en la homogeneidad de la microestructura y aparecen zonas concretas con grandes defectos. Por tanto, la aplicación tecnológica de estos recubrimientos es muy limitada en estas condiciones, debido a la aparición de grietas y poros, pero sí aportan información valiosa, en cuanto a la formación de la microestructura del recubrimiento.

\subsection{Análisis microestructural}

La microestructura de ambos recubrimientos es semejante, apareciendo tres zonas claramente diferenciadas cuya extensión depende de la velocidad de enfriamiento.

\subsubsection{Zona central}

En la parte central del recubrimiento, aparece una acumulación de carburos de gran tamaño. Las dimensiones de los bloques de WC, disminuyen con la cercanía a la interfase o la superficie externa (Fig. 5). Presentan una morfología facetada, formada por grandes caras planas y que es semejante a la de los carburos primarios que contiene el polvo, aunque su tamaño es mayor que en el resto del recubrimiento. Esto sugiere, que se produce un fuerte engrosamiento durante el procesado por láser. En esta zona, la velocidad de enfriamiento es menor y las fuerzas de Marangoni son más ineficientes para realizar el movimiento efectivo del material no fundido ${ }^{[14]}$. Por tanto, hay menor cantidad de elementos de aleación provenientes del substrato y el proceso de dilución y formación de estructuras complejas está menos activado.

Alrededor de esta banda de carburos, aparecen pequeñas lagunas en las que se han disuelto los carburos (det 1, Fig. 5). En su interior aparecen microestructuras de tipo eutéctico con una fina dispersión de carburos en su interior (Fig. 6). El análisis de la composición en diversos puntos marcados en esta figura (Tabla 2), muestra la presencia de elementos de aleación procedentes del sustrato de acero, como el hierro y el cromo. Este hecho es importante, puesto que el análisis se ha tomado en la zona superior del cordón, e indica que hay una gran difusión de los elementos de aleación del sustrato que es potenciada por las fuerzas convectivas del material fundido. Esta estructura es típica en recubrimientos de base $\mathrm{Ni}$-Cr-Fe con un contenido inferior al $30 \%$ en $\mathrm{W}^{[7,9}$ y 11$]$, en los que el cromo y el hierro, actúan como activadores del proceso de disolución del WC a temperaturas inferiores a las esperadas.

Para comprobar estas hipótesis, se han tomado diferentes espectros EDX en la zona, a distancia

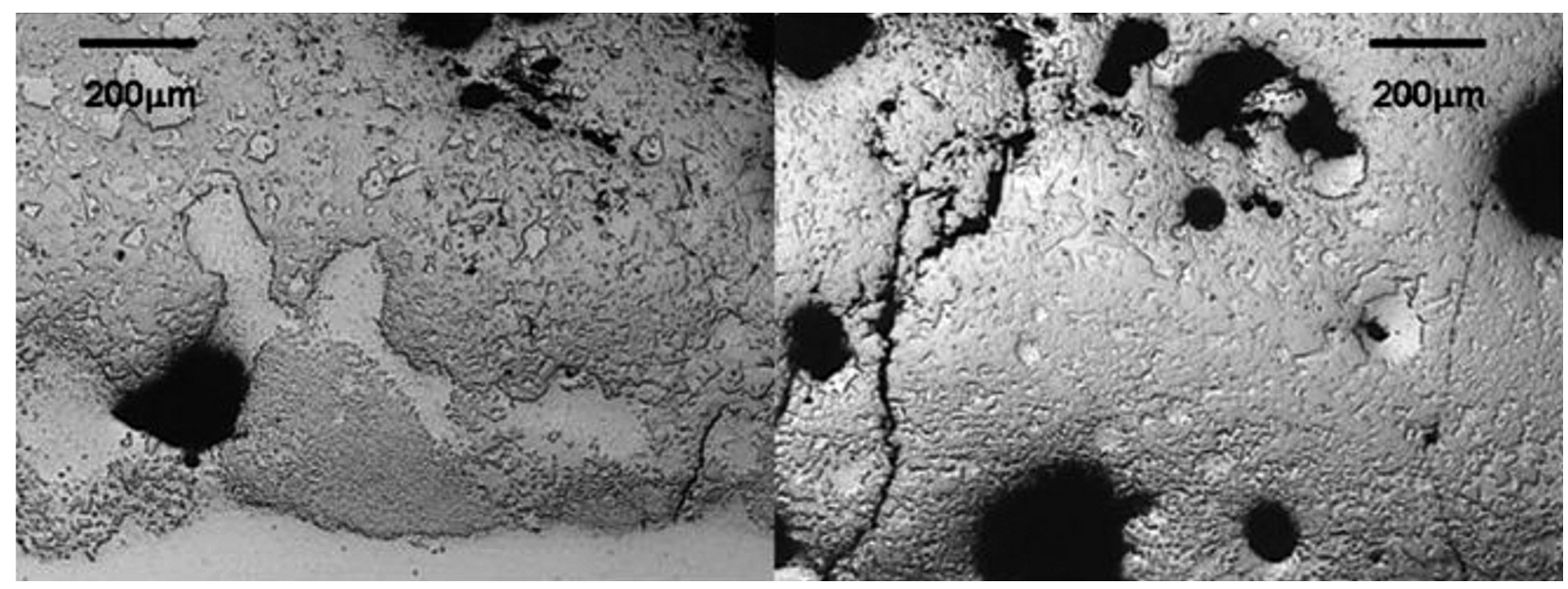

Figura 4. Efecto de la potencia $(P)$ y velocidad $(V)$, en la formación de defectos en los recubrimiento $1(P=400 \mathrm{~W}, \mathrm{~V}=250 \mathrm{~mm} / \mathrm{min})$ y recubrimiento $2(\mathrm{P}=500 \mathrm{~W}, \mathrm{~V}=375 \mathrm{~mm} / \mathrm{min})$.

Figure.4. Effect of power $(P)$ and velocity $(V)$, in the formation of defects in the coating $1(P=400 \mathrm{~W}$, $V=250 \mathrm{~mm} / \mathrm{min})$ and coating $2(P=500 \mathrm{~W}, V=375 \mathrm{~mm} / \mathrm{min})$. 


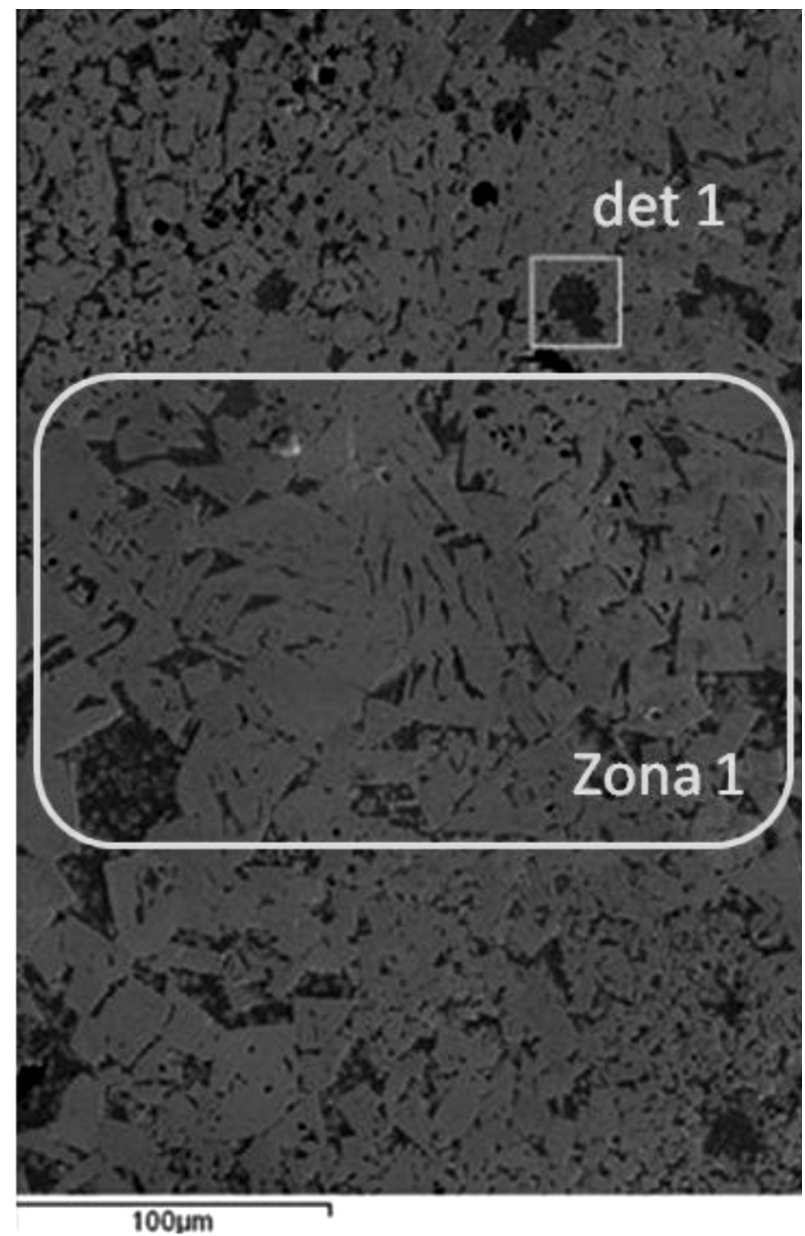

Figura 5. Engrosamiento del WC en la zona central del recubrimiento 1 (zona 1). Además se detalla la ubicación del detalle de la figura 6 .

Figure 5. Coarsening of the WC in the central area of coating 1 (zone 1). Besides detailing the location of the detail of figure 6 .

suficiente para evitar el efecto del solape. El espectro 1 muestra la composición semejante a la original de un carburo primario no disuelto, si bien hay una cierta disminución del wolframio que ha migrado hacia la

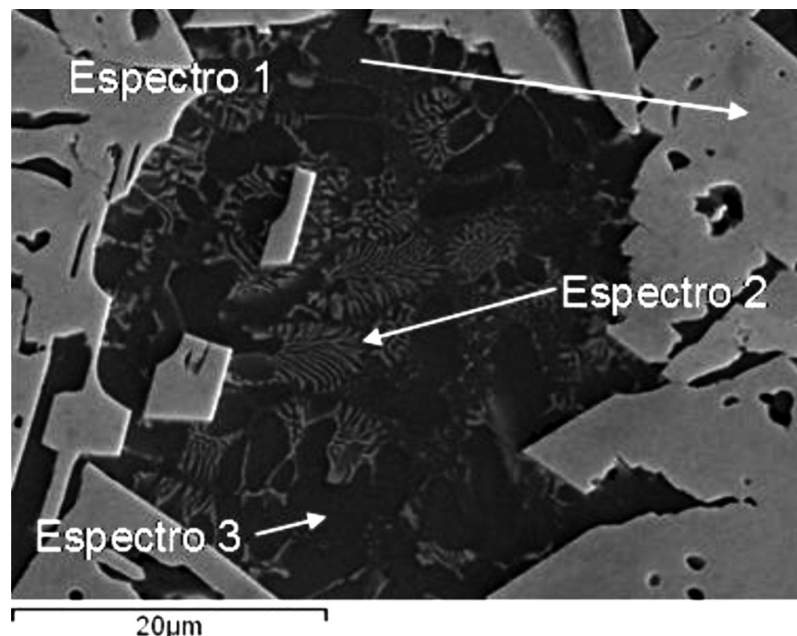

Figura 6. Detalle 1 que muestra una laguna de dilución en la parte superior del recubrimiento y detalla los puntos, donde se obtuvieron los microanálisis por EDS de la tabla 2.

Figure 6. Detail 1 which shows a gap at the top dilution of the coating and details the points which were obtained by EDS microanalysis in table 2.

laguna. Sin embargo, el espectro 2, que recoge la composición alrededor del eutéctico, muestra una cantidad importante de hierro. La microestructura es extremadamente compleja ya que el sistema $\mathrm{Ni}$ $\mathrm{Cr}-\mathrm{Fe}-\mathrm{W}$, puede presentar diversos eutécticos en función de la composición.

En esta zona aparecen precipitados carburos compuestos de (wolframio, cromo, níquel y hierro) muy pequeños, que no han sido identificados ${ }^{[7,8 \text { y } 13]}$. No obstante, su morfología, bajo contenido en wolframio $\mathrm{y}$ alto contenido en hierro, sugieren que se trata de una mezcla de carburos (WC, $\mathrm{W}_{2} \mathrm{C}$ y del tipo M6C) ${ }^{[8]}$. Finalmente, en el espectro 3, se muestra la composición de un área formada principalmente por $\gamma-\mathrm{Ni}$, saturado por los diferentes elementos de aleación en solución.

Tabla 2. Resumen de la composición encontrada en los diferentes análisis

Table 2. Summary of the composition found in the different analysis

\begin{tabular}{lrrrrrrrrrr}
\hline & \multicolumn{2}{c}{$\mathbf{w}$} & \multicolumn{2}{c}{$\mathbf{C}$} & \multicolumn{2}{c}{$\mathbf{N i}$} & \multicolumn{2}{c}{$\mathbf{F e}$} & \multicolumn{2}{c}{$\mathbf{C r}$} \\
& $\mathbf{\%} \mathbf{w}$ & $\mathbf{\%} \mathbf{a}$ & $\mathbf{\%} \mathbf{w} \mathbf{\%}$ & $\mathbf{a}$ & $\mathbf{\%} \mathbf{w}$ & $\mathbf{\%} \mathbf{a}$ & $\mathbf{\%} \mathbf{w}$ & $\mathbf{\%} \mathbf{a}$ & $\mathbf{\%} \mathbf{w}$ & $\mathbf{\%} \mathbf{a}$ \\
\hline Espectro 1 & 82,03 & 22,97 & 17,97 & 77,03 & & & & & & \\
Espectro 2 & 26,56 & 5,79 & 15,94 & 53,22 & 19,95 & 13,36 & 30,36 & 21,81 & 7,25 & 5,55 \\
Espectro 3 & 11,07 & 2,44 & 13,11 & 44,3 & 53,92 & 37,27 & 20,46 & 14,86 & 1,44 & 1,13 \\
Espectro 4 & 65,92 & 19,98 & 12,61 & 58,48 & 2,76 & 2,62 & 15,17 & 15,13 & 3,54 & 3,7 \\
\hline
\end{tabular}




\subsubsection{Zona inferior}

En la parte inferior del recubrimiento las partículas de WC, son de menor tamaño, con formas más afiladas que sugieren diferentes tipos de carburos que se han formado debido al procesado por láser (Figs. 7B) y 7C)). Se ha comprobado que existe una gran difusión de elementos de aleación en ambas direcciones, especialmente de hierro y cromo hacia el recubrimiento y de wolframio hacia el sustrato de acero, siendo su contenido mayor en las lagunas de dilución, al igual que se indica en la figura 6.

Debido a la mayor velocidad de enfriamiento, en el interior de las lagunas aparecen formaciones dendríticas, según el flujo del calor ${ }^{[7]}$, formadas principalmente por una fina estructura eutéctica de carburos de wolframio, en una matriz de níquel. Y se ha comprobado que pequeñas variaciones en la cantidad de wolframio y cromo presente, modifican la estructura, ya que el Ni-Cr favorece la formación de eutécticos muy finos ${ }^{[15]}$.

\subsubsection{Zona cercana a la interfase}

Finalmente, en esta zona aparecen compuestos con una morfología totalmente diferente. Estas estructuras, suelen aparecer en los aceros rápidos refundidos superficialmente por láser que han sufrido múltiples pasadas, en los que se ha provocado varias fusiones del material $^{[11]}$. Especialmente hay que destacar la formación de dos tipos de compuestos: uno en forma de agujas (Fig. $7 \mathrm{C})$ ), que no han podido ser identificados con precisión debido a su pequeño tamaño, pero que podrían ser de tipo complejo $\mathrm{M}_{23} \mathrm{C}_{6}$ o $\mathrm{M}_{34} \mathrm{C}_{10}$ y otro con forma poligonal ligeramente redondeada (Fig. 8). Estos últimos se caracterizan por un alto contenido en wolframio y por presentar un corazón con un con-

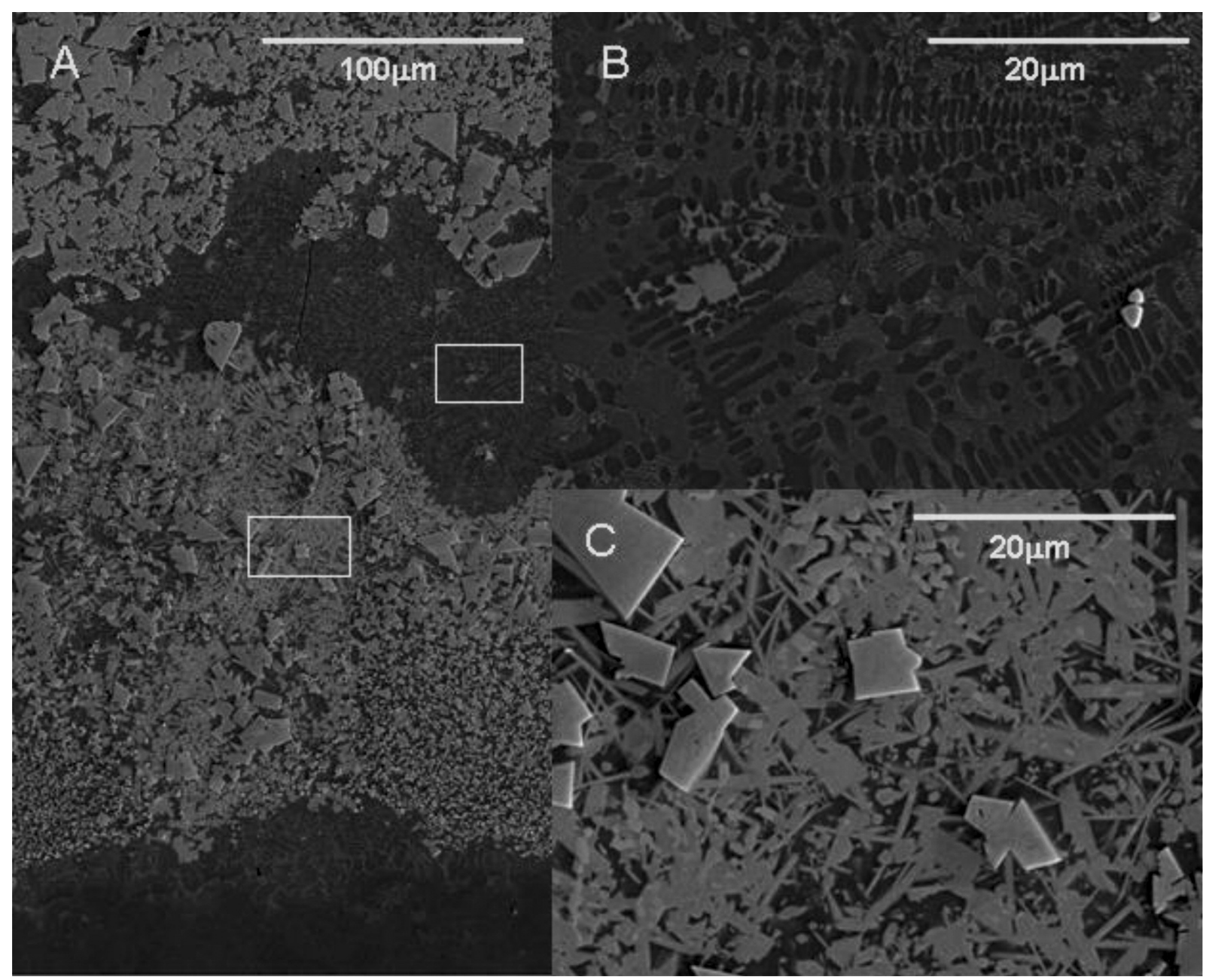

Figura 7. Microestructura de la zona inferior del recubrimiento y detalles de la formación de compuestos.

Figure 7 Microstructure of the lower cladding area and details of the formation of compounds. 


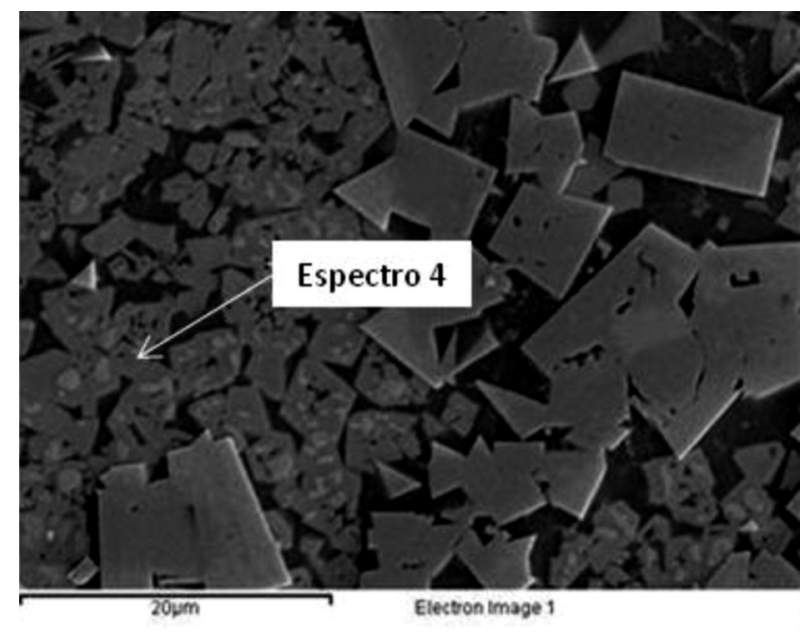

Figura 8. Imagen en BSE de los compuestos redondeados en la zona inferior.

Figure 8. Image of the rounded compounds in $B S E$ at the bottom.

tenido en wolframio mayor, posiblemente formado por $\mathrm{W}_{2} \mathrm{C}$ y una cobertura del tipo $\mathrm{M}_{6} \mathrm{C}^{[8]}$. En el espectro 4 (Tabla 2), se recoge su composición.

\section{EVOLUCIÓN DE LA DUREZA}

Aunque se han tomado tres medidas alrededor de cada punto, con la misma distancia a la interfase. Debido a la heterogeneidad de la microestructura, no se encuentran diferencias significativas entre ambos recubrimientos. Sin embargo, en el sustrato se aprecia un endurecimiento mayor en la zona cercana a la interfase, para el recubrimiento con mayor velocidad de enfriamiento, debido a la mayor cantidad de martensita formada, figura 9. Por tanto, una reducción excesiva de la velocidad de enfriamiento, reduce el efecto del templado por láser sobre el acero con un efecto perjudicial.

Es importante destacar el efecto endurecedor de la difusión de wolframio hacia el sustrato en la zona de fusión de éste, incrementando la dureza desde los $790 \mathrm{HV}$ útiles para este acero, hasta los $850 \mathrm{HV}$ para el recubrimiento 2. En ambos recubrimientos, el mayor pico de la dureza se produce allí donde hay mayor cantidad de carburos complejos, que han precipitado a partir del líquido formado ${ }^{[17]}$ y que tienen forma de agujas. Por tanto, se confirma la naturaleza dura de estas fases. Por último, se aprecia un descenso en la dureza en la zona central, donde se produce un engrosamiento de los carburos.

\section{CONCLUSIONES}

- El procesado láser de recubrimientos compuestos de $\mathrm{WC}+\mathrm{Ni}$, exige un control estricto de la energía aportada para evitar la dilución parcial o total de las partículas de WC.

- La velocidad de enfriamiento es un parámetro crítico para evitar el agrietamiento de los recubrimientos.

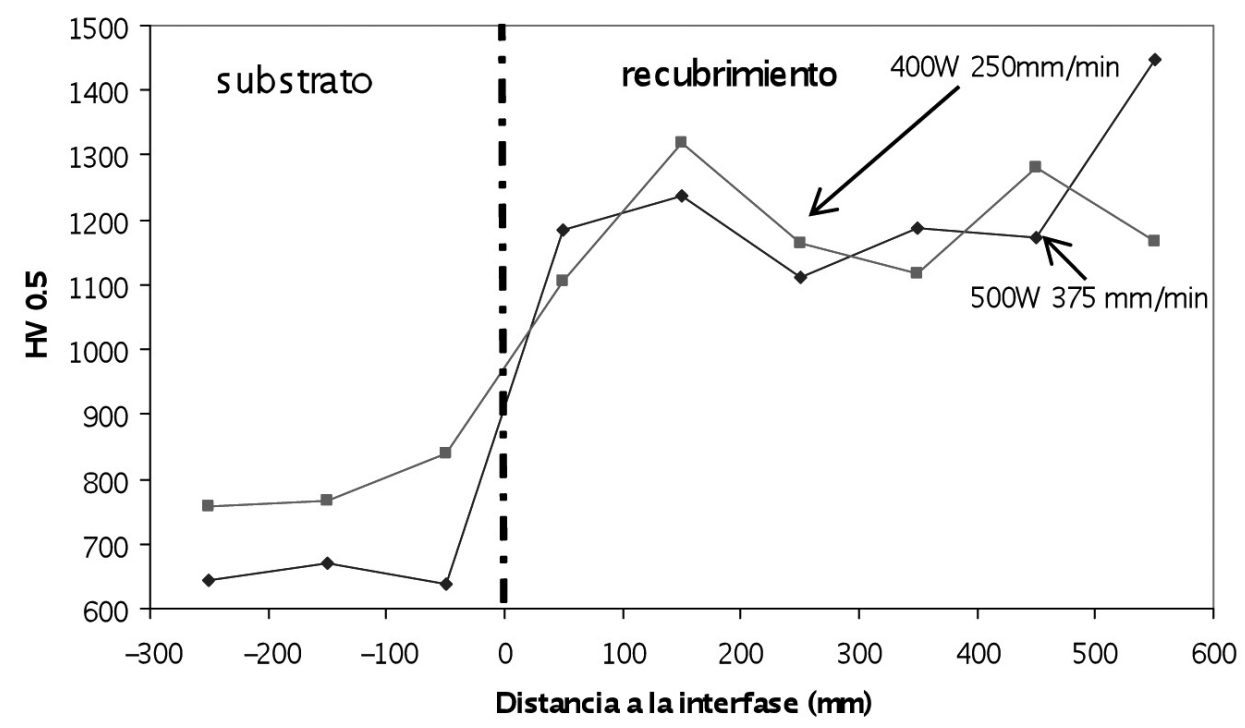

Figura 9. Evolución de la dureza obtenida en los recubrimientos.

Figure 9. Evolution of the hardness obtained in coatings. 
- Para la ventana de parámetros y par de materiales estudiados, ha sido inevitable la formación de porosidad e irregularidades en la deposición de recubrimientos de $\mathrm{WC10Ni}$, por solape sin precalentamiento del sustrato.

- Existe una ventana de proceso, en la cual se puede evitar la dilución de las partículas de WC, consiguiendo que permanezcan con su morfología original, aunque aparece un cierto engrosamiento de los carburos en la zona central del recubrimiento.

- Cuando se reduce la velocidad de enfriamiento, aparece una gran difusión de los elementos de aleación en ambos sentidos de hierro y cromo desde el sustrato hacia el recubrimiento, llegando incluso a su parte superior y de wolframio hacia el sustrato.

- La microestructura formada en las lagunas de dilución es muy compleja debido al sistema $\mathrm{Ni}-\mathrm{Fe}-\mathrm{Cr}-\mathrm{W}$ que forma eutécticos complejos extremadamente finos.

- Aparecen diferentes tipos de compuestos de pequeño tamaño del tipo $\mathrm{WC}, \mathrm{W}_{2} \mathrm{C}, \mathrm{M}_{6} \mathrm{C}$ y $\mathrm{M}_{23} \mathrm{C}_{6}$ con una elevada dureza, que aumenta la fragilidad del recubrimiento y que condiciona las propiedades finales.

\section{REFERENCIAS}

[1] H. Zhang, G. Wang, Y. Luo y T. Nakaga, Thin Solid Films 390 (2001) 7-12.

[2] P. Cadenas, M. Rodriguez, y M. H. Staia, Rev. Metal. Madrid 43 (2007) 50-62.
[3] R. Vilar, J. Laser. Appl. 11, 64-79.

[4] I. Vicario, C. Soriano, C. Sanz, R. Bayón y J. Leunda, Rev. Metal. Madrid 45 (2009) 14-19.

[5] Z. Chen, L. Lim y M. Qian, J. Mater. Process. Tech. 62 (1996) 321-323.

[6] M.J. Tobar, C. Álvarez, J.M. Amado, G. Rodríguez y A. Yáñez, Surf. Coat. Tech. 200 (2006) 6.313-6.317.

[7] L.C. Lim, Q. Ming y Z. Chen, Surf. Coat. Tech. 106 (1998) 183-192.

[8] P. Wang, J. Qu y H. Wang, Mater. Design, 17 (1996) 289-296.

[9] A. Hidouci, J.M. Pelletier, F. Ducoin, D. Dezert y R. Guerjouma, Surf. Coat. Tech. 123 (2000) 17-23.

[10] Y. Xianqing, Z. Chengjun y S. Xuefeng, Appl. Surf. Sci. 253 (2007) 4.409-4.414.

[11] M. Riabkina y E. Rabkin, J. Mater. Sci. letters 20(2001) 1.917-1.920.

[12] S.W. Huang, M. Samandi y M. Brandt, Wear 256 (2004) 1.095-1.105.

[13] B.S. Sidhu y D. Puri, J. Mater. Proc. Tech. 159 (2005) 347-355.

[14] L. St-Georges, Wear 263 (2007) 562-566.

[15] J. Przybylowiz, J. Mater. Proc. Tech. 109 (2001) 154-160.

[16] P. Wu, H.M. Du, X.L. Chen, Z.Q. Li y H.L. Bai, Wear 257 (2004) 142-147.

[17] M. Cadenas, J. M. Cuetos, J. E. Fernández, M.R. Fernández, L. M. Vega y J. de Damborenea, Rev. Metal. Madrid 38 (2002) 457-463. 